\title{
Baixa fecundidade e adiamento do primeiro filho no Brasil
}

\author{
Adriana Miranda-Ribeiro* \\ Ricardo Alexandrino Garcia* \\ Tereza Cristina de Azevedo Bernardes Faria ${ }^{\star \star *}$
}

\begin{abstract}
O Brasil vem passando por um processo de adiamento dos nascimentos, o que tem contribuído para que a fecundidade observada seja reduzida pela ação do efeito tempo. Nesse contexto, o primeiro nascimento assume importância, na medida em que o momento da sua ocorrência está relacionado ao dos nascimentos subsequentes e ao potencial de recuperação dos nascimentos adiados. 0 objetivo deste trabalho é analisar o comportamento dos nascimentos de primeira ordem no Brasil, levando-se em conta a heterogeneidade regional. Buscam-se elementos que possam enriquecer o debate acerca do futuro da fecundidade no país. São utilizados os dados dos Censos Demográficos e as histórias de nascimentos reconstruídas a partir destes, para o período de 1980 a 2010. Os resultados mostram a persistência dos diferenciais regionais e indicam um cenário de adiamento do primeiro filho no Brasil e de aumento da proporção de mulheres que terminam o período reprodutivo sem filhos. Caso os diferenciais regionais se reduzam, a tendência é de acirramento do adiamento e queda adicional dos níveis de fecundidade.
\end{abstract}

Palavras-chave: Adiamento da fecundidade. Nascimentos de primeira ordem. Brasil.

\footnotetext{
* Centro de Desenvolvimento e Planejamento Regional (Cedeplar), Universidade Federal de Minas Gerais (UFMG), Belo Horizonte-MG, Brasil (adrianamr@cedeplar.ufmg.br; https://orcid.org/0000-0002-6845-7926).

** Instituto de Geociências (IGC), Universidade Federal de Minas Gerais (UFMG), Belo Horizonte-MG, Brasil (alexandrinogarcia@gmail.com; https://orcid.org/0000-0001-7144-9866).

*** Centro de Desenvolvimento e Planejamento Regional (Cedeplar), Universidade Federal de Minas Gerais (UFMG), Belo Horizonte-MG, Brasil (tbernardesfaria@gmail.com; https://orcid.org/0000-0001-7199-3888).
} 


\section{Introdução e objetivos}

A queda continuada da fecundidade no Brasil coloca em destaque alguns questionamentos sobre o seu futuro. Quais os níveis mínimos que serão alcançados? Haverá queda adicional, estabilização da fecundidade em baixos níveis ou recuperação para níveis mais próximos ao da reposição? Existe uma consonância entre demógrafos de que os baixos níveis de fecundidade são uma consequência direta do adiamento dos nascimentos (GOLDSTEIN; SOBOTKA; JASILIOLIENE, 2009). Nesse sentido, a discussão sobre o futuro da fecundidade não pode estar dissociada dessa temática.

A queda da fecundidade de coorte é um dos fatores citados como consequência do adiamento prolongado dos nascimentos (KOHLER; ORTEGA, 2002; GOLSDSTEIN; LUTZ; SCHERBOV, 2004; MYRSKYLÄ; KOHLER; BILLARI, 2009). A relação entre os dois fenômenos não é direta. Quando há postergação dos nascimentos em um período, a fecundidade de coorte pode se manter constante caso as mulheres recuperem os nascimentos adiados, chegando ao final do período reprodutivo com o número de filhos que teriam na ausência do adiamento. No entanto, se os nascimentos adiados não são totalmente recuperados, a fecundidade de coorte será afetada, assim como a de período (KOHLER; ORTEGA, 2002).

A não recuperação dos nascimentos adiados depende de uma série de fatores, sendo que um deles está relacionado à idade da mulher, que impacta a fertilidade. Leridon (2004) estima que cerca de $18 \%$ das mulheres são incapazes de engravidar após os 36 anos de idade, aumentando para $40 \%$ após os 40 anos e para $90 \%$ após os 45 anos. Nesse sentido, quanto mais longo for o adiamento, menor será a chance de recuperação.

0 potencial de recuperação dos nascimentos adiados está relacionado, também, à ordem do nascimento adiado. 0 momento do nascimento do primeiro filho é determinante para o comportamento reprodutivo futuro da mulher, uma vez que a idade ao primeiro filho está associada à idade aos nascimentos subsequentes (SIMÃO et al., 2006). 0 adiamento do primeiro nascimento pode, também, ser um fator contribuinte para o aumento da proporção de mulheres que terminam o período reprodutivo sem filhos. A ausência de filhos - ou childlessness - tem crescido nos países desenvolvidos e, mais recentemente, também no Brasil (ALVES; CAVENAGHI; BARROS, 2010; MENEGHIM; OLIVEIRA, 2016; MENEGHIM, 2018; LEOCÁDIO, 2018). Kohler e Ortega (2002) apontam que, caso a transição para o primeiro filho seja adiada por conta do adiamento de outras transições (educação ou casamento/ coabitação, por exemplo), poderá haver consequências sobre o nível de mulheres sem filhos ao final do período reprodutivo, indicando, no mínimo, alteração na fecundidade final de coorte.

Essa discussão é permeada pelo que Lutz, Skirbekk e Testa (2006) denominam como armadilha demográfica da baixa fecundidade. Os autores chamam a atenção para a importância da estrutura etária da população no debate sobre baixa fecundidade, adiamento e recuperação da fecundidade. Embora níveis baixos de fecundidade reduzam o número de nascimentos, o crescimento populacional é garantido se a população tiver uma estrutura 
etária jovem. No entanto, longos períodos de baixa fecundidade geram uma estrutura etária envelhecida, com um número decrescente de mulheres entrando no período reprodutivo a cada momento. Nesse cenário, nem mesmo uma eventual recuperação da fecundidade livraria a população do decrescimento.

0 presente trabalho pretende contribuir para o debate sobre baixos níveis de fecundidade e seu futuro no Brasil, analisando o adiamento do primeiro filho. Desse modo, o objetivo principal é abordar, a partir de uma perspectiva regional, a tendência e o comportamento dos nascimentos de primeira ordem nas últimas décadas. Além disso, almeja-se compreender os diferentes ritmos em que as transformações ocorreram nas cinco grandes regiões e em quais pontos as tendências se divergem ou se assemelham. Também espera-se entender as mudanças na proporção de filhos de primeira ordem, além de detectar como o efeito tempo tem atuado em cada região.

Para tanto, foram utilizados os microdados dos Censos Demográficos de 1980 a 2010 e as histórias de nascimentos reconstruídas a partir dos Censos de 1991 a 2010. As bases de dados foram empregadas para estimar as medidas tradicionais de fecundidade, além daquelas não tradicionais, como as derivadas da aplicação de modelo de decomposição da variação da fecundidade e as razões de progressão por parturição (PPR).

\section{Adiamento da fecundidade no Brasil}

0 adiamento da fecundidade é caracterizado pelo aumento da idade média da fecundidade entre dois períodos (BONGAARTS; FEENEY, 1998; ORTEGA; KOHLER, 2002; BONGAARTS; SOBOTKA, 2012). Nesse caso, o efeito tempo é positivo e a fecundidade corrente é diminuída pela redução do número de nascimentos no período. 0 fenômeno pode ocorrer em uma ou mais ordens de nascimento e sempre altera a fecundidade em relação ao nível que seria observado na sua ausência (BONGAARTS; FEENEY, 1998; ORTEGA; KOHLER, 2002; BONGAARTS; SOBOTKA, 2012).

No Brasil, a fecundidade está abaixo do nível de reposição desde meados da década de 2000. Entre 2000 e 2010, também, as variações na idade média da fecundidade indicaram um processo de adiamento em curso. Isso indica que a queda da fecundidade observada no país tem sido intensificada pela postergação dos nascimentos (MIRANDA-RIBEIRO; RIOS-NETO; GARCIA, 2016). O comportamento verificado para o total do país contrasta com aquele observado para grupos distintos. Diante disso, Miranda-Ribeiro, Rios-Neto e Ortega (2006) estimaram o efeito tempo segundo raça/cor e escolaridade, utilizando dados dos Censos Demográficos de 1991 e 2000. Os resultados apontam que as mulheres brancas e aquelas com maior nível de escolaridade (4-7 anos de estudo) estavam, nos anos 1980 e 1990 , mais próximas do adiamento da fecundidade do que as negras e aquelas com menor escolaridade (0-3 anos de estudo). No final da década de 1990, as mulheres brancas eram as únicas, dentre as categorias analisadas, a adiar o nascimento do primeiro filho. 
Os dois grupos mais próximos do adiamento apresentaram, também, níveis inferiores de fecundidade e maior proporção de nascimentos de ordens 1 e 2.

Ao incluir na análise da fecundidade o Censo Demográfico de 2010, Miranda-Ribeiro e Garcia (2013) apontam que o Brasil é um país de múltiplas transições. Os autores analisam indicadores de fecundidade das mulheres brasileiras segundo o nível de escolaridade, para o período de 1980 a 2010 . Os resultados mostram que o país, como um todo, parecia estar terminando a (primeira) transição demográfica. No entanto, a análise por nível educacional indica que os grupos de 0-7 anos de estudo e de 12 anos e mais de estudo encontravam-se em situações opostas: enquanto as mais escolarizadas mostravam indícios de estarem passando pela segunda transição demográfica, as menos escolarizadas encontravam-se ainda na primeira fase. 0 estudo sugere declínio adicional da fecundidade, por conta da crescente escolarização das mulheres. Nesse caso, esperava-se que, ao menos em parte, as mulheres assumissem um novo comportamento reprodutivo, à medida que adquirissem ganhos em escolaridade. Só isso já seria motivo para queda adicional da fecundidade. Assim, o futuro da fecundidade dependeria da trajetória - e principalmente da velocidade desta - que cada grupo educacional traçaria dali em diante.

Rios-Neto et al. (2018) decompõem medidas de fecundidade segundo a escolaridade da mãe para determinar em que extensão a queda da fecundidade no Brasil foi determinada pelo efeito taxa ou pelo efeito composição. A abordagem teórica traz dois modelos. No primeiro, a queda da fecundidade seria explicada pela convergência das taxas observadas para os diversos grupos educacionais, prevalecendo o efeito taxa. No segundo modelo, as diferenças de fecundidade entre grupos educacionais se manteriam constantes e a queda da fecundidade se daria pelas mudanças na composição da população feminina segundo os estratos educacionais, prevalecendo o efeito composição. Os resultados indicam que a transição da fecundidade no Brasil ocorreu em função tanto da mudança da composição por educação quanto da queda da fecundidade dentro dos grupos educacionais. Com relação aos níveis futuros, os autores apontam que se - e enquanto - os níveis de fecundidade dos grupos de menor escolaridade continuarem caindo, os efeitos taxa e composição atuarão em conjunto. Caso os níveis de fecundidade desses grupos se estabilizem, o efeito composição passará a predominar.

Não há estudos no Brasil que apontem a extensão do adiamento ou mesmo uma eventual recuperação futura dos níveis de fecundidade. No entanto, as projeções populacionais da Organização das Nações Unidas - ONU - (UN, 2017) indicam que a fecundidade no Brasil deverá atingir 1,65 filho por mulher em 2050/2055 e, a partir de então, apresentará uma recuperação, chegando a 1,77 filho por mulher em 2095/2100. As projeções mais recentes do IBGE (2018) mostram queda gradativa, alcançando 1,66 filho por mulher em 2060.

Independentemente da recuperação - ou não - dos nascimentos, é fato que, no Brasil, de modo geral, o fenômeno do adiamento dos nascimentos tem contribuído para que a fecundidade observada seja diminuída, em relação a um valor hipotético - aquele que seria verificado na ausência do adiamento. Nesse contexto, os primeiros nascimentos 
têm papel fundamental. Goldstein (2006) aponta que, em um cenário no qual mais do que a terça parte dos primeiros nascimentos ocorra entre mulheres com idade superior a 35 anos, há um impacto significativo no número de mulheres que chegam ao final do período reprodutivo sem filhos, contribuindo para a intensificação e manutenção dos baixos níveis de fecundidade. Em 2017, 10\% dos nascimentos de primeira ordem no Brasil ocorreram de mulheres com 35 anos ou mais de idade (ABDO, 2019). Esse percentual coloca o país ainda distante do cenário extremo, mas é preciso pensar que, para além do aumento do percentual das mulheres sem filhos, o adiamento da maternidade também acarreta o crescimento da proporção de mulheres que chegam ao final do período reprodutivo com um filho. Nos dois casos, pode-se inferir que fica mais distante a possibilidade de retomada da fecundidade para níveis próximos ao da reposição.

\section{Bases de dados}

Nesse trabalho, foram utilizados os microdados dos Censos Demográficos de 1980, 1991, 2000 e 2010, disponibilizados pelo Instituto Brasileiro de Geografia e Estatística (IBGE). Taxas e idade média da fecundidade, percentuais de nascimentos e medidas ajustadas foram calculadas diretamente dos microdados das quatro edições do Censo, a partir das variáveis total de nascidos vivos e total de nascidos vivos no ano anterior, segundo a idade da mãe. Para as razões de progressão por parturição, foram considerados os microdados das histórias de nascimentos reconstruídas a partir dos Censos de 1991, 2000 e 2010, o que permitiu a construção de uma série histórica de indicadores para o período 1980 a 2010.

\section{Histórias de nascimentos reconstruídas a partir de Censos Demográficos}

A metodologia de reconstrução de histórias de nascimentos baseada no processo de pareamento, descrita em Miranda-Ribeiro, Rios-Neto e Carvalho (2009), utiliza informações sobre a estrutura domiciliar/familiar e algumas variáveis sobre reprodução, disponíveis no Censo, para alocar no tempo os nascimentos declarados das mulheres recenseadas. Além de alocar os filhos presentes no domicílio, imputam-se as idades dos filhos omitidos ausentes ou falecidos -, por um período de 15 anos anteriores a cada Censo. No banco de dados final, cada linha corresponde a uma mulher e contém variáveis que indicam quantos filhos ela teve em cada ano e a ordem dos filhos nascidos, nos 15 anos anteriores ao Censo. Essas informações permitem a construção de uma série histórica anual de indicadores de fecundidade. 0 encadeamento de histórias de nascimentos reconstruídas a partir das edições dos Censos de 1991, 2000 e 2010 fornece uma série histórica de indicadores para o período de 1977 a 2010.

A metodologia aplicada visa completar as histórias de nascimento de mulheres, nos 15 anos anteriores ao Censo ou pesquisa utilizada. Em termos gerais, o processo consiste em procurar, dentre as histórias de nascimentos completas, a que mais se assemelha a 
uma história parcial de nascimentos, baseada na comparação de diversas variáveis sociodemográficas. Diante disso, a história de nascimentos completa que possuir a relação mais forte com a incompleta determina as variáveis que serão imputadas posteriormente (MIRANDA-RIBEIRO, 2007; MIRANDA-RIBEIRO; RIOS-NETO; CARVALHO, 2009).

0 procedimento consiste em: alocar crianças a mães dentro do domicílio/família; determinar as histórias de nascimento completas e incompletas, construindo dois conjuntos de dados; parear conjuntos de histórias de nascimento (completos e incompletos) e determinar a melhor correspondência; e imputar a idade dos filhos omitidos (MIRANDA-RIBEIRO, RIOS-NETO; CARVALHO, 2009). O banco de dados final gerado com as histórias de nascimento é composto por todas as mulheres recenseadas - mulheres sem filhos, aquelas que originalmente tiveram histórias completas de nascimento, as que tiveram suas histórias de nascimento imputadas e aquelas que não tiveram suas histórias de nascimento imputadas devido à ausência de um par - para que seja possível calcular as medidas relacionadas à fecundidade. Embora o procedimento possa ser aplicado a todas as mulheres que respondem aos quesitos sobre reprodução (a partir de dez anos de idade), optou-se por limitar às mulheres de 15 a 64 anos de idade na data de referência do Censo. ${ }^{1}$ Com isso, o banco de dados contém informações completas de mulheres de 15 a 49 anos para todo o período.

As vantagens de se utilizar uma história de nascimento obtida por meio da manipulação dos dados fornecidos pelos Censos Demográficos estão relacionadas: ao tamanho da amostra, que permite a desagregação geográfica e por características socioeconômicas e demográficas; à precisão dos dados, assegurando a robustez dos resultados; e ao número de edições nas quais as metodologias podem ser aplicadas, o que proporciona a criação de uma série temporal longa e contínua (MIRANDA-RIBEIRO; RIOS-NETO; CARVALHO, 2007; RIOS-NETO; MIRANDA-RIBEIRO; MIRANDA-RIBEIRO, 2018).

O banco de dados das histórias de nascimentos reconstruídas se distingue do resultante da aplicação de outros métodos conhecidos, como o método dos filhos próprios (MFP) (CHO; RETHERFORD; CHOE, 1986), cujos dados são trabalhados no nível agregado, não permitindo, dentre outras coisas, identificar a ordem dos nascimentos ocorridos a cada ano. 0 MFP possibilita o cálculo de taxas de fecundidade específicas e totais, enquanto as histórias de nascimentos permitem o cálculo dessas taxas, também, por ordem de nascimento, além de outras medidas baseadas em probabilidade (MIRANDA-RIBEIRO, 2007; MIRANDA-RIBEIRO; RIOS-NETO; CARVALHO, 2009).

Para checar a qualidade das informações, comparam-se as séries históricas de taxas de fecundidade total geradas pelas histórias de nascimentos com aquelas produzidas por outros métodos. Miranda-Ribeiro (2007) mostra que a qualidade da série gerada pelas histórias de nascimentos é satisfatória para um período de até dez anos. Assim, neste

\footnotetext{
${ }^{1}$ Optou-se, também, por não incluir as mulheres de 10 a 14 anos de idade, uma vez que essas não são consideradas no cálculo da taxa de fecundidade total, mantendo-se, assim, a comparabilidade dos indicadores.
} 
trabalho, são apresentadas medidas derivadas das histórias de nascimentos para o período de 1980 a 2010.

\section{Medidas relativas ao primeiro nascimento}

Percentual de nascimentos, taxa de fecundidade total, taxas específicas de fecundidade relativas e idade média da fecundidade

Ao longo do tempo, a queda da parturição provocou aumento do peso relativo dos nascimentos de primeira ordem no Brasil. De acordo com dados dos Censos Demográficos, em 1980 , os primeiros nascimentos correspondiam a $26,9 \%$ do total de nascimentos, percentual que aumentou para 34,1\% em 1991, 39,6\% em 2000 e 45,1\% em 2010 (Tabela 1). Esse crescimento foi acompanhado por variações na idade média da fecundidade da primeira ordem de nascimento $\left(M_{A C}\right)$. Em 1980, as brasileiras tiveram o primeiro filho, em média, aos 23,5 anos de idade. No entanto, até 2000, houve declínio da $M A C_{1}$, que atingiu 23,1 anos em 1991 e 22,9 anos em 2000. Por sua vez, na década seguinte, houve reversão do declínio e a $M A C_{1}$ chegou a 24,0 anos em 2010 . As taxas de fecundidade total de primeira ordem, que consideram nos seus cálculos apenas os nascimentos de primeira ordem em cada ano, declinaram $11 \%$ entre 1980 e 2010 . A redução mais acentuada ocorreu entre 1980 e 1991, mas foi também significativa entre 2000 e 2010.

TABELA 1

Proporção de nascimentos de primeira ordem, taxa de fecundidade total (TFT 1 ) e idade média da fecundidade $\left(M_{1} C_{1}\right)$

Brasil e regiões - 1980-2010

\begin{tabular}{|c|c|c|c|c|c|c|c|}
\hline Variáveis & Anos & Brasil & Norte & Nordeste & Sudeste & Sul & $\begin{array}{l}\text { Centro- } \\
\text {-Oeste }\end{array}$ \\
\hline \multirow{4}{*}{$\begin{array}{l}\text { Proporção de nascimentos de } \\
1 \stackrel{\text { a }}{\text { ordem }}\end{array}$} & 1980 & 26,9 & 20,2 & 20,0 & 32,1 & 32,2 & 26,7 \\
\hline & 1991 & 34,1 & 26,6 & 28,6 & 39,3 & 38,8 & 38,5 \\
\hline & 2000 & 39,6 & 33,7 & 37,5 & 42,0 & 40,5 & 42,2 \\
\hline & 2010 & 45,1 & 38,0 & 44,2 & 47,5 & 46,6 & 44,5 \\
\hline \multirow{4}{*}{$\begin{array}{l}\text { Idade média da fecundidade, } 1 \text { a } \\
\text { ordem de nascimento }\left(M^{\prime} C_{1}\right)\end{array}$} & 1980 & 23,5 & 22,3 & 23,4 & 23,9 & 23,3 & 22,7 \\
\hline & 1991 & 23,1 & 21,8 & 22,9 & 23,6 & 23,0 & 22,5 \\
\hline & 2000 & 22,9 & 21,5 & 22,6 & 23,5 & 23,0 & 22,4 \\
\hline & 2010 & 24,0 & 22,3 & 23,4 & 24,8 & 24,5 & 23,6 \\
\hline \multirow{4}{*}{$\begin{array}{l}\text { Taxa de fecundidade total, } 1 \underline{\text { a }} \\
\text { ordem de nascimento }\left(\mathrm{TFT}_{1}\right)\end{array}$} & 1980 & 0,952 & 0,941 & 0,931 & 0,948 & 0,964 & 0,962 \\
\hline & 1991 & 0,885 & 0,875 & 0,866 & 0,855 & 0,914 & 0,946 \\
\hline & 2000 & 0,882 & 0,935 & 0,902 & 0,849 & 0,871 & 0,914 \\
\hline & 2010 & 0,844 & 0,892 & 0,880 & 0,807 & 0,818 & 0,852 \\
\hline
\end{tabular}

Fonte: IBGE. Censos Demográficos 1980, 1991, 2000 e 2010 (microdados). 
Entre as regiões brasileiras, foram encontrados comportamentos distintos (Tabela 1). No Norte, observam-se as menores proporções de nascimentos de primeira ordem em todos os períodos e a menor idade média da fecundidade para nascimentos de primeira ordem do país. 0 Nordeste registra o maior aumento na proporção de nascimentos de primeira ordem: em 1980, encontrava-se em nível inferior ao verificado no Norte, contudo, em 2010, atingiu nível próximo ao das regiões Centro-Oeste, Sul e Sudeste. A MAC 1 declina a partir de 1980 e aumenta entre 2000 e 2010, permanecendo abaixo do Sudeste e Sul. As regiões Sudeste e Sul apresentam comportamentos semelhantes. Em 1980, o percentual de nascimentos de primeira ordem já era superior a 30\%. Ao final do período analisado, mais de $45 \%$ dos nascimentos nessas regiões eram de primeiros filhos. Com relação à $M A C_{1}$, as regiões registram os maiores valores no período e são as primeiras a indicar a reversão da queda (Tabela 1 ).

O Gráfico 1 apresenta as taxas específicas de fecundidade relativas, para primeiros nascimentos, no Brasil e nas regiões, para o período 1980-2010.

Para o Brasil, as mulheres de 20 a 24 anos são responsáveis pela maior proporção de nascimentos de primeira ordem em todos os períodos, mas há mudanças significativas no padrão. Em 1980 e 1991, cerca de 45\% dos nascimentos de primeira ordem foram de mulheres desse grupo etário. No entanto, em 2000, o grupo perdeu em participação para o grupo de 15 a 19 anos. Nos três primeiros períodos, as mulheres acima de 30 anos mantiveram constante a participação relativa nos nascimentos de primeira ordem. Em 2010, houve queda da participação relativa dos grupos 15-19 e 20-24 anos (sendo maior a do primeiro) e aumento da participação dos grupos etários acima dos 30 anos.

Já a região Norte apresenta comportamento distinto das demais regiões, mostrando maior concentração dos nascimentos de primeira ordem entre as mulheres de 15 a 19 anos em 1991, 2000 e 2010; em 1980, a diferença entre esse grupo e o de 20 a 24 anos foi a menor, comparando com as demais regiões.

No Nordeste, o comportamento das taxas específicas de fecundidade relativas é semeIhante ao do Brasil. Destacam-se, no entanto, a maior participação das mulheres do grupo 15 a 19 e 20 a 24 anos, em todos os períodos, e a menor participação daquelas acima de 30 anos, quando comparadas ao observado para o país.

No Sudeste e no Sul, as mulheres de 20 a 24 anos concentram o maior percentual de nascimentos de primeira ordem em todos os períodos. Nessas regiões, destaca-se 0 afastamento da curva referente a 2010 para mulheres entre 25 e 39 anos de idade, indicando que houve crescimento da participação relativa dessas mulheres na composição da $\mathrm{TEF}_{1}$. Esse afastamento também é evidente no Centro-Oeste. 0 que chama a atenção nesta região em relação ao Sudeste e Sul é a maior participação das mulheres de 15 a 19 anos em 2000. 


\section{GRÁFICO 1}

Taxas específicas de fecundidade relativas, para nascimentos de primeira ordem Brasil e regiões - 1980-2010
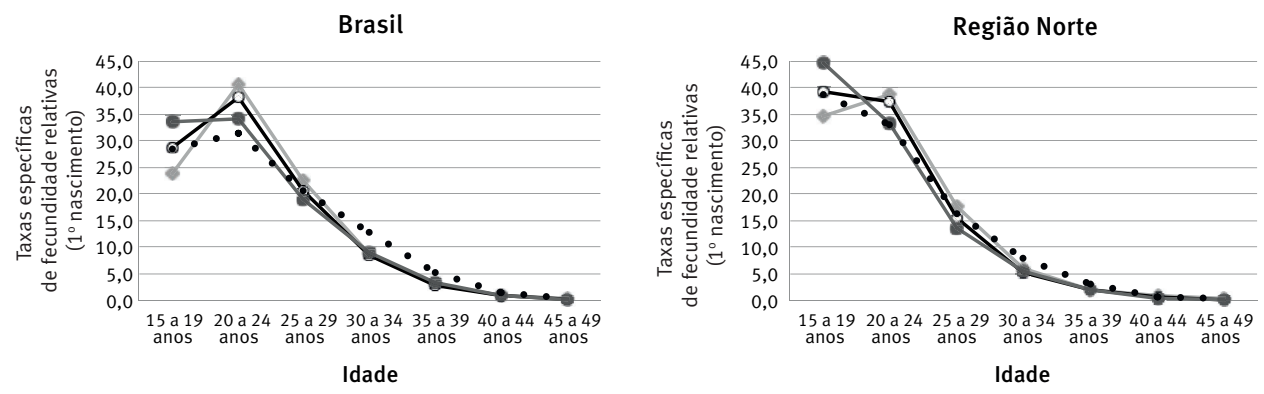

Região Nordeste

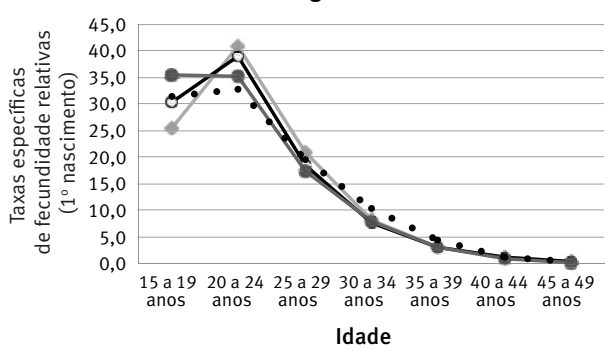

Região Sudeste
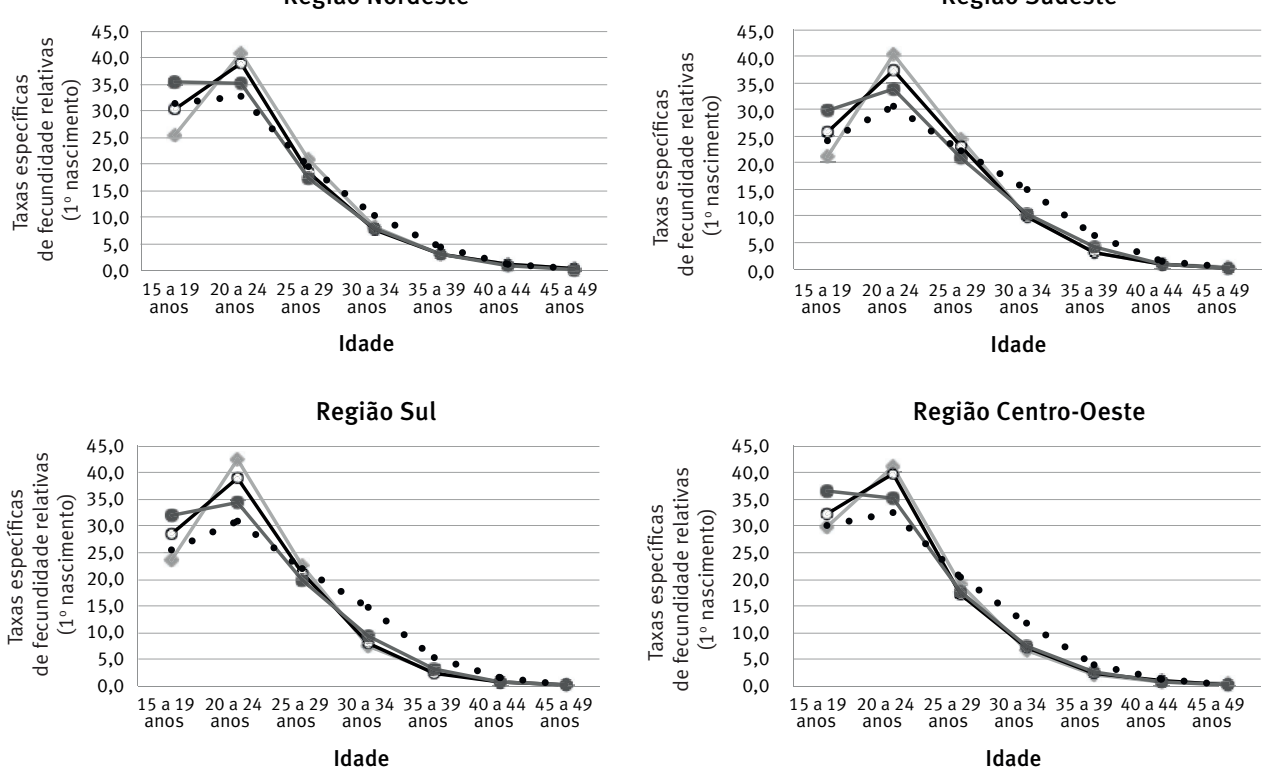

$\rightarrow 1980 \multimap 1991 \rightarrow 2000 \quad \cdots 2010$

Fonte: IBGE. Censos Demográficos 1980, 1991, 2000 e 2010 (microdados).

\section{Efeito tempo e razão de progressão por parturição (PPR)}

Efeito tempo segundo o modelo BF

No modelo de Bongaarts e Feeney (1998), efeito tempo está relacionado a distorções que variações na idade média da fecundidade causam na TFT observada em cada período. 0 modelo tem como objetivo calcular uma nova TFT, livre das distorções causadas pelo efeito tempo. Essa medida, denominada TFT ajustada, representa a TFT que seria observada na ausência das mudanças na idade média da fecundidade, isto é, na ausência do efeito tempo. A TFT ajustada é a medida que Bongaarts e Feeney (1998) definem como o quantum puro. 
Os autores assumem que a fecundidade é influenciada pela idade, parturição, período, duração desde o nascimento anterior, mas não por coorte. Um argumento para o desenvolvimento do modelo BF é que as mudanças na fecundidade durante um certo período podem ocorrer em qualquer idade ou ordem de nascimento, como uma consequência tanto do quantum quanto do efeito tempo. Assim, o modelo trabalha considerando a ordem de nascimento e utiliza uma adaptação da equação desenvolvida por Ryder (1956), que calcula a TFT ajustada. Além de permitir a estimativa do efeito tempo para a TFT, permite que se estime o efeito tempo que atua em cada ordem de nascimento.

As duas principais críticas ao modelo BF referem-se ao fato de os autores desconsiderarem os diferenciais de coorte na mudança da idade média da fecundidade e usarem medidas inadequadas na aplicação do modelo (VAN IMHOFF; KEILMAN, 2000). A equação 1 demonstra como o ajuste é feito.

$\operatorname{TFT}_{i, \text { ajustada }}=\frac{\operatorname{TFT}_{i, \text { observada }}}{\left(1-r_{i}\right)}$

Onde: $T F T_{i, \text { observada }}$ é a taxa de fecundidade total observada para a ordem de nascimento $i$; e $r_{i}$ é a variação anual da idade média da fecundidade para a ordem de nascimento $i$ (medida em anos). A $T F T_{\text {observada }}$ é calculada como a soma das $T F T_{i, \text { observada }}$ e a $T F T_{\text {ajustada }}$ é obtida pela soma das $T F T_{i, \text { ajustada }}$, para cada ordem $i$ de nascimento. Neste trabalho, foram calculadas as $T F T_{1}$ (primeira ordem de nascimento) observada e ajustada. Para o cálculo das $r_{i}$ são necessários sempre dois períodos consecutivos e a $T F T_{i, \text { ajustada }}$ é sempre calculada para o final do período. Os dados foram retirados dos Censos Demográficos de 1980 a 2010.

A Tabela 2 apresenta os resultados (apenas para nascimentos de primeira ordem) da aplicação do modelo BF (1998) para 1991, 2000 e 2010. Os resultados levam em conta a variação da idade média da fecundidade para a ordem de nascimento no período anterior, isto é, entre 1980 e 1991, entre 1991 e 2000 e entre 2000 e 2010, respectivamente. A TFT $_{\text {ajustada }}$ indica a taxa que seria observada para a primeira ordem de nascimento, na ausência de mudanças na idade média da fecundidade dessa ordem. Quando a ajustada é maior do que a observada, há adiamento dos nascimentos, contudo, quando a observada é maior do que a ajustada, pode-se inferir que houve antecipação.

Os resultados indicam que, entre 1980 e 1991, os nascimentos de primeira ordem foram antecipados no Brasil em todas as regiões, gerando uma $T F T_{1, \text { observada }}$ superior à que realmente seria na ausência de queda da $M A C_{1}$. Os maiores valores para a $T F T_{1, \text { observada }}$ ocorreram no Sul e Centro-Oeste e os menores no Sudeste. A região Sul foi a primeira a adiar nascimentos de primeira ordem, o que aconteceu entre 1991 e 2000. Nesse mesmo período, os resultados apontam que no Sudeste os nascimentos de primeira ordem ainda eram antecipados, embora a diferença entre as $T F T_{1}$ observada e ajustada tenha sido pequena. No período seguinte, entre 2000 e 2010, os resultados mostram adiamento do primeiro nascimento em todas as regiões brasileiras. 
TABELA 2

Taxas de fecundidade total observadas e ajustadas (modelo BF) para a primeira ordem de nascimento Brasil e regiões - 1991-2010

\begin{tabular}{clcccccc}
\hline Anos & Variáveis & Brasil & Norte & Nordeste & Sudeste & Sul & $\begin{array}{c}\text { Centro- } \\
\text {-Oeste }\end{array}$ \\
\hline 1991 & TFT $T_{1, \text { observada }}$ & 0,885 & 0,875 & 0,866 & 0,855 & 0,914 & 0,946 \\
& TFT $T_{1, \text { ajustada }}$ & 0,848 & 0,826 & 0,824 & 0,824 & 0,884 & 0,928 \\
& Efeito tempo & Antecipação & Antecipação & Antecipação & Antecipação & Antecipação & Antecipação \\
\hline 2000 & TFT $T_{1, \text { observada }}$ & 0,882 & 0,935 & 0,902 & 0,849 & 0,871 & 0,914 \\
& TFT $T_{1, \text { ajustada }}$ & 0,860 & 0,902 & 0,868 & 0,840 & 0,873 & 0,894 \\
& Efeito tempo & Antecipação & Antecipação & Antecipação & Antecipação & Adiamento & Antecipação \\
\hline 2010 & TFT $T_{1, \text { observada }}$ & 0,844 & 0,892 & 0,880 & 0,807 & 0,818 & 0,852 \\
& TFT $T_{1, \text { ajustada }}$ & 0,962 & 0,987 & 0,970 & 0,943 & 0,971 & 0,986 \\
& Efeito tempo & Adiamento & Adiamento & Adiamento & Adiamento & Adiamento & Adiamento \\
\hline
\end{tabular}

Fonte: IBGE. Censos Demográficos 1980, 1991, 2000 e 2010 (microdados).

Razão de progressão por parturição (parity progression ratios - PPR)

A razão de progressão por parturição (PPR) indica a proporção de mulheres que progridem de uma parturição, $i$, para a parturição seguinte, $i+1$. No caso da PPR da primeira ordem de nascimento, a proporção é de mulheres que têm o primeiro filho.

As progressões podem ser obtidas a partir de dados de coorte ou de período. Os cálculos de coorte geralmente usam dados de censos ou pesquisas sobre o número de crianças já nascidas, classificadas por idade da mãe (ou por duração do casamento, quando há a informação - o que não é o caso dos censos brasileiros). Os cálculos de período são feitos usando probabilidades de nascimento específicas por parturição.

A maior limitação do uso de medidas de coorte é que se referem a um período distante no tempo. No entanto, mesmo o comportamento de mulheres que terminaram - ou estão próximas de terminar - seu período reprodutivo pode fornecer indícios de comportamento futuro das mulheres. Ao mesmo tempo, as PPR de mulheres mais jovens podem evidenciar algumas tendências, mesmo que haja a possibilidade de novas progressões, uma vez que ainda não terminaram seu período reprodutivo.

A relação entre $\mathrm{M}(\mathrm{i})$, mulheres com pelo menos i filhos, e as $\mathrm{M}(\mathrm{i}+1)$, mulheres com pelo menos $i+1$ filhos, indica a proporção de mulheres que progridem para a parturição $\mathrm{i}+1$, sendo que a(i) é dada por:

$a(i)=\frac{M(i+1)}{M(i)}$

Em geral, consideram-se coortes de mulheres no cálculo, isto é, as PPR são calculadas a partir da distribuição por parturição de um grupo particular de mulheres. Para as mulheres que finalizaram o período reprodutivo, as medidas são fixas; para aquelas que ainda se encontram no período reprodutivo, existe a possibilidade de progressão para a parturição seguinte. Neste trabalho, foram calculadas as PPR de período. A série histórica de período indica o comportamento reprodutivo de diversas coortes de mulheres. Para o cálculo da 
PPR para o primeiro filho, a(0), utilizam-se, no numerador, o número de mulheres que têm pelo menos um filho e, no denominador, todas as mulheres.

O Gráfico 2 mostra os valores de a(0), calculados para mulheres de 45-49, 40-44, 35-39 e 30-34 anos de idade, entre 1980 e 2010, para Brasil e regiões. Os dados foram retirados das histórias de nascimentos, que permitem estimativas para períodos intercensitários. Os valores indicam a proporção de mulheres dessas coortes que tiveram o primeiro filho. De modo geral, observa-se que as diversas regiões apresentam níveis distintos de PPR. No entanto, a tendência de diminuição do percentual de mulheres que progridem para o primeiro filho, representada pela queda da a(0) ao final do período, é semelhante.

Por estarem no final do período reprodutivo, os resultados obtidos para as mulheres de 45-49 anos de idade são tidos como definitivos, ainda que uma pequena parcela dessas mulheres possa, eventualmente, progredir para o primeiro filho. A queda da progressão para essas mulheres indica aumento do percentual daquelas que terminam o período reprodutivo sem filhos. Estão representadas oito coortes de mulheres, que nasceram entre os quinquênios 1931-1935 e 1961-1965 e que em 1980, 1985, etc. tinham 45-49 anos de idade.

Mulheres de 40-44 anos de idade estão caminhando para o final do período reprodutivo, embora haja uma maior chance de progredirem entre as parturições, em comparação às mulheres de 45-49 anos. As curvas referentes a esse grupo representam as mulheres nascidas entre 1936-1940 e 1966-1970 e que tinham 40-44 anos de idade no período 1980 a 2010.

Mulheres de 35-39 anos nasceram entre 1941-1945 e 1971-1975 e as de 30-34 anos nasceram entre 1946-1950 e 1976-1980. Elas representam coortes de mulheres que ainda estão relativamente distantes do final do período reprodutivo e, portanto, com mais tempo para a progressão. Apesar da distância, seu comportamento com relação à progressão para o primeiro filho pode trazer boas reflexões sobre o futuro da fecundidade.

Para o Brasil, a proporção de mulheres acima de 40 anos que tiveram o primeiro filho está próxima de $90 \%$ durante boa parte do período. A partir de 2000 , há queda e, ao final do período, observa-se que aproximadamente 87\% das mulheres de 40-44 e 45-49 anos de idade foram mães. Até 1995, cerca de 90\% de mulheres de 35-39 anos de idade havia tido o primeiro filho; a partir de então, observa-se queda e, ao final do período, por volta de $85 \%$ das mulheres dessa idade tornaram-se mães. Para as mulheres de 30 34 anos, a proporção das que progrediram para o primeiro filho é mais baixa: não passa de $85 \%$ no início do período e, a partir de 1995, decresce, até atingir valores próximos de $75 \%$ em 2010 .

Na região Norte, os resultados indicam a maior proporção de mulheres que tiveram o primeiro filho, para todas as idades. Até 2000 , mais de $90 \%$ das mulheres dos grupos etários 35-39 a 45-49 anos haviam progredido para o primeiro filho. Entre as mais jovens, a proporção é próxima de $90 \%$ até 1995 e, a partir daí, inicia-se a queda, chegando a 83\% em 2010. Mulheres de 40-44 e 45-49 anos apresentam comportamento semelhante. 


\section{GRÁFICO 2}

Razões de progressão por parturição, primeiro filho, $a(0)$, segundo grupos de idade Brasil e regiões - 1980-2010
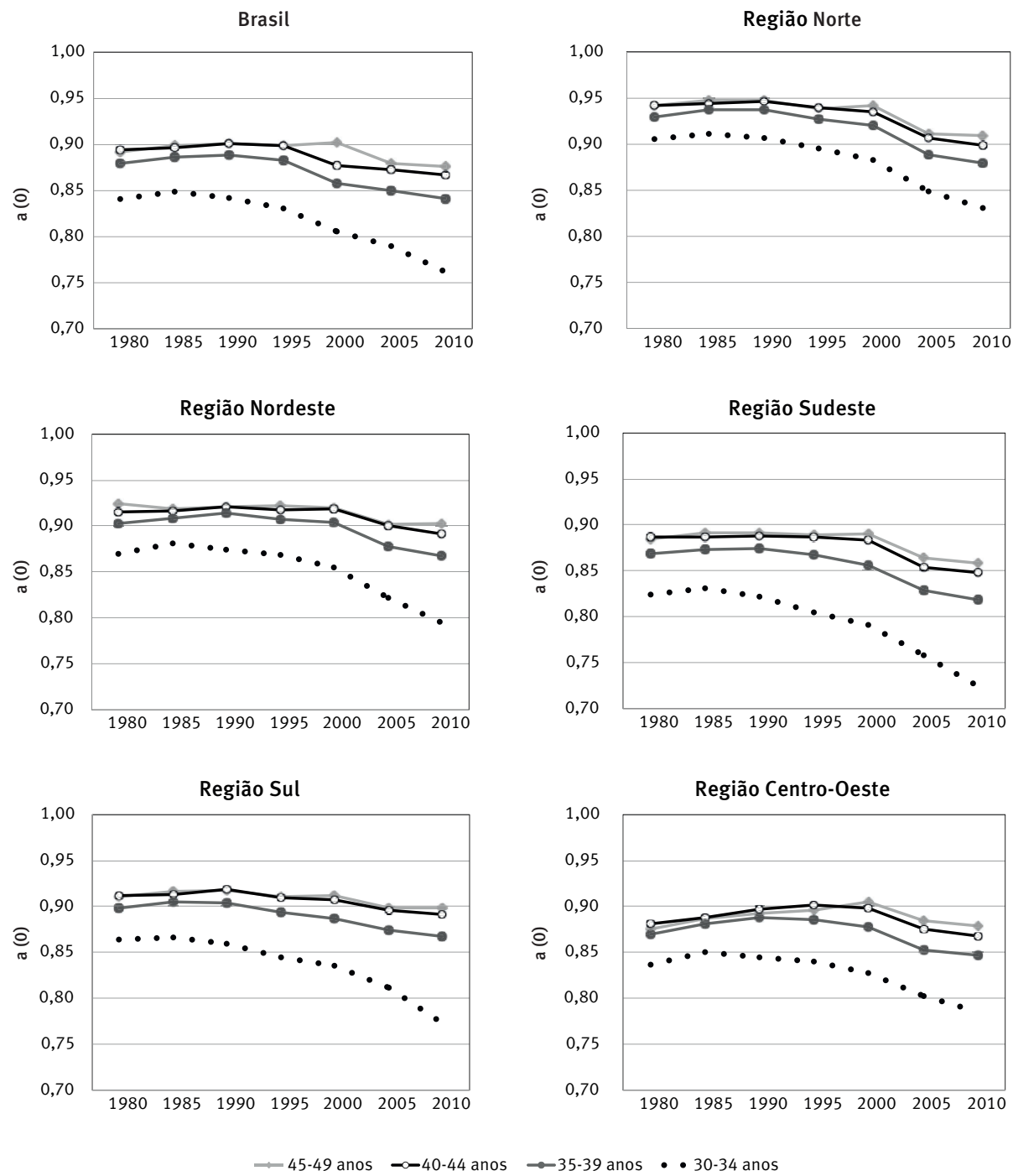

Fonte: IBGE. Censos Demográficos de 1991, 2000 e 2010 (histórias de nascimentos reconstruídas).

No Nordeste, mais de $90 \%$ das mulheres de 35-39 a 45-49 anos haviam tido o primeiro filho entre 1980 e 2000. A partir daí, observa-se declínio nas proporções. Ao final do período, as proporções de mulheres de 35-39, 40-44 e 45-49 anos com ao menos um filho correspondiam a, respectivamente, $90 \%, 89 \%$ e $87 \%$. Entre as mais jovens, o percentual daquelas que progrediram era superior a $85 \%$ até 2000 , chegando a aproximadamente $80 \%$ em 2010. 
As menores proporções de mulheres que progrediram para o primeiro filho, em todas as idades, estão no Sudeste. Aquelas de 40-44 e 45-49 anos têm comportamento semeIhante e menos de $90 \%$ tinham progredido para o primeiro filho, já em 1980.0 percentual permanece constante até 2000, quando declina para valores próximos de 85\%, em 2010. Dentre as mulheres de 35-39 anos, uma proporção próxima de $87 \%$ havia progredido até 1995; em 2010, 83\% das mulheres dessa idade tinham tido o primeiro filho.

A região Sul tem alto percentual de mulheres de 40-44 e 45-49 anos que progrediram para o primeiro filho, mesmo ao final do período analisado, próximo dos $90 \%$. A progressão para o primeiro filho é também alta entre as mulheres de 35-39 anos. 0 declínio da progressão é maior para as mais jovens, de 30-34 anos, cuja proporção é de 86\% em 1980 e $77 \%$ em 2010.

No Centro-Oeste, o comportamento em relação à progressão para o primeiro filho é distinto do restante do país. Para mulheres de 40-49 anos, observa-se que a progressão aumentou de $87 \%$ para $90 \%$, entre 1980 e 2000 , com posterior declínio, voltando ao nível inicial. Entre as mulheres de 35-39 anos, a progressão cresceu até 2000 e depois diminuiu, alcançando $85 \%$ em 2010. Para as mulheres mais jovens, observa-se queda na proporção das que tiveram o primeiro filho, entre 1985 e 2000 , de $85 \%$ para $79 \%$.

\section{Discussão e conclusão}

0 adiamento do primeiro nascimento implica mudanças que vão além da redução da fecundidade observada em cada período, na medida em que afetam progressões subsequentes e podem fazer com que as mulheres cheguem ao final do período reprodutivo com um número menor de filhos do que teriam na sua ausência. Embora a taxa específica de fecundidade da primeira ordem de nascimento venha caindo, a crescente participação dos primeiros nascimentos na composição da fecundidade torna essa análise importante. 0 presente artigo teve como objetivo analisar o adiamento do primeiro nascimento no Brasil no contexto da heterogeneidade regional e os resultados encontrados trouxeram alguns elementos para se pensar o futuro da fecundidade no país.

Todos os resultados apontam para um cenário de adiamento do primeiro filho no Brasil, em todas as regiões analisadas, em maior ou menor grau. 0 aumento da idade média ao primeiro filho ocorreu na região Sul, entre 1991 e 2000, acompanhada pelo restante do país, entre 2000 e 2010 . No Norte, o aumento da $M A C_{1}$ foi o mais discreto. Além disso, entre 2000 e 2010 , a região também apresentou a menor queda da participação relativa das mulheres jovens (15-19 anos) e o menor aumento da participação relativa das mulheres mais maduras, na composição da $\mathrm{TEF}_{1}$. Nas regiões Sul, Sudeste e Centro-Oeste, a queda da participação das mulheres de 15-19 anos nos nascimentos de primeira ordem foi maior. Ademais, o distanciamento da curva das TEF relativas em 2010 indica que a maior participação das mulheres de 30-39 anos na fecundidade de primeira ordem é bastante evidente. 
0 comportamento das PPRs também foi diferenciado entre as regiões. Analisando-se os resultados para as mulheres de 40-44 e 45-49 anos, já no final do período reprodutivo, a queda da progressão para o primeiro filho está em acordo com o aumento das mulheres childless na população brasileira. Se a tendência de queda das PPRs permanecer e/ou se houver convergência no comportamento reprodutivo entre as regiões, o cenário futuro é de intensificação da childlessness no país. Para as mulheres de 30-34 e 35-39 anos, a queda das PPR para o primeiro nascimento não necessariamente indica que chegarão ao final do período reprodutivo sem filhos, pois elas ainda têm um relativo tempo para progredirem. Contudo, caso essas mulheres - ou parte delas, ao menos - progridam, isso significa que os declinantes percentuais de progressão nessas idades estão relacionados ao adiamento da maternidade. A não progressão, por sua vez, indica o adiamento sem recuperação.

Os diferenciais encontrados confirmam que o comportamento de todas as mulheres brasileiras não é uniforme e que diferentes fatores vão impactar as transformações da fecundidade. Além dos diferenciais educacionais, fica claro que o processo de transição da fecundidade é impactado também pelos diferenciais regionais. No entanto, as mudanças constatadas nas regiões Norte e Nordeste permitem inferir que estas tendem a seguir 0 caminho das demais, possibilitando uma convergência futura nas tendências para o adiamento do primeiro filho. Um dos indícios é que, entre os Censos de 2000 e 2010, todas as regiões apresentaram sinais de postergamento.

Este trabalho não teve a pretensão de projetar ou discutir uma eventual recuperação dos nascimentos adiados, mas almejou levantar elementos que servissem como base para essa discussão e ajudassem a pensar em futuros caminhos para a fecundidade no país. Para além dos níveis de fecundidade, devem-se ter em conta as consequências - que podem ser irreversíveis - decorrentes de longos períodos em baixos níveis. No Brasil, esse pode ser um problema a ser enfrentado no futuro, se persistirem os baixos níveis, associados ao adiamento dos nascimentos, em especial do primeiro filho.

\section{Referências}

ABDO, M. P. M. Adiamento e gravidez de risco: associação entre idade materna avançada com baixo peso e prematuridade dos filhos. Dissertação (Mestrado) - Universidade Federal de Minas Gerais, Belo Horizonte, 2019.

ALVES, J. E. D.; CAVENAGHI, S. M.; BARROS, L. F. W. A família DINC no Brasil: algumas características sociodemográficas. Rio de Janeiro: Escola Nacional de Ciências Estatísticas, IBGE, 2010. (Textos para discussão, n. 30).

BILLARI, F.; KOHLER, H-P. Patterns of lowest-low fertility in Europe. Rostok, Germany: Max Planck Institute for Demographic Research, 2002. (Working Paper 2002-040).

BONGAARTS, J.; SOBOTKA, T. A demographic explanation for the recent rise in European fertility. Population and Development Review, v. 38, n. 1, p. 83-120, 2012.

BONGAARTS, J. Demographic consequences of declining fertility. Science, v. 282, n. 5388, p. 419-420, 1998. 
BONGAARTS, J.; FEENEY, G. On the quantum and tempo of fertility. Population and Development Review, v. 24, n. 2, p. 271-291, 1998.

BRASS, W. Methods for estimating fertility and mortality from limited and defective data. Chapel Hill, NC: The North Carolina Center, 1975.

CHO, L.-J.; RETHERFORD, R. D.; CHOE, M. K. The own-children method of fertility estimation. Honolulu, Hawaii: University of Hawaii, 1986.

DAVIS, K.; BLAKE, J. Social structure and fertility: an analytic framework. Economic Development and Cultural Change, v. 4, n. 3, p. 211-235, 1956.

GOLDSTEIN, J. How late can first births be postponed? Some illustrative population-level calculations. Vienna Yearbook of Population Research, v. 4, n. 1, p. 153-165, 2006.

GOLDSTEIN, J.; LUTZ, W.; SCHERBOV, S. Long-term population decline in Europe: the relative importance of tempo effects and generational length. Population and Development Review, v. 29, n. 4, p. 699-707, 2003.

GOLDSTEIN, J. R.; SOBOTKA, T.; JASILIONIENE, A. The end of lowest-low fertility? Population and Development Review, v. 35, n. 4, p. 663-700, 2009.

KOHLER, H.-P.; ORTEGA, J. A. Tempo-adjusted period parity progression measures, fertility postponement and completed cohort fertility. Demographic Research, v. 6, n. 6, p. 91-144, 2002.

LEOCÁDIO, V. A. Childlessness no Brasil: a contribuição das mudanças sociodemográficas para a tendência de zero filho. Dissertação (Mestrado) - Universidade Federal de Minas Gerais, Belo Horizonte, 2018.

LERIDON, H. Can assisted reproduction technology compensate for the natural decline in fertility with age? A model assessment. Human Reproduction, v. 19, n. 7, p. 1548-1553, 2004.

LESTHAEGHE, R. The second demographic transition: a concise overview of its development. Proceedings of the National Academy of Sciences, v. 111, n. 51, p. 18112-18115, 2014.

LUTZ, W.; SKIRBEKK, V.; TESTA, M. R. The low-fertility trap hypothesis: forces that may lead to further postponement and fewer births in Europe. Vienna Yearbook of Population Research, v. 4, n. 1, p. 167-192, 2006.

MENEGHIM, J. C. A.; OLIVEIRA, M. C. F. A. Análise da relação entre ausência de filhos e características socioeconômicas e demográficas para mulheres de 50 a 59 anos no período de 1980 a 2010 no Brasil. In: ENCONTRO NACIONAL DE ESTUDOS POPULACIONAIS, 20., 2016, Foz do Iguaçu/PR. Anais [...]. Belo Horizonte: Abep, 2016.

MENEGHIM, J. C. A. Mulheres sem filhos no Brasil: uma análise de características socioeconômicas e demográficas, razões e repercussões. Tese (Doutorado) - Universidade Estadual de Campinas, Campinas, 2018.

MIRANDA-RIBEIRO, A. Reconstrução de histórias de nascimentos a partir de dados censitários: aspectos teóricos e evidências empíricas. Tese (Doutorado) - Universidade Federal de Minas Gerais, Belo Horizonte, 2007.

MIRANDA-RIBEIRO, A.; RIOS-NETO, E. L. G.; CARVALHO, J. A. M. Reconstrução de histórias de nascimentos a partir de dados censitários: uma análise comparativa de duas metodologias. Revista Brasileira de Estudos de População, Rio de Janeiro, v. 26, n. 1, p. 21-35, 2009.

MIRANDA-RIBEIRO, A.; GARCIA, R. A. Transition or transitions? Analyzing the fertility decline in Brazil in the light of educational levels. Revista Latinoamericana de Población, v. 7, n. 13, p. 91-106, 2013. 
MIRANDA-RIBEIRO, A.; RIOS-NETO, E. L. G.; ORTEGA, J. A. Efeito tempo, quantum e efeito parturição na transição da fecundidade no Brasil: aplicação do modelo de Kohler \& Ortega. In: ENCONTRO NACIONAL DE ESTUDOS POPULACIONAIS, 15., 2006, Caxambu/MG. Anais [...]. Caxambu: Abep, 2006.

MIRANDA-RIBEIRO, A.; RIOS-NETO, E. L. G.; GARCIA, R. A. Anticipación y postergación de los nacimientos en la transición de la fecundidad en Brasil. Notas de Población (Impresa), v. 103, p. 29-43, 2016.

MYRSKYLÄ, M.; KOHLER, H.-P.; BILLARI, F. C. Advances in development reverse fertility declines. Nature, v. 460, p. 741-743, 2009.

ORTEGA, J. A.; KOHLER, H.-P. Measuring low fertility: rethinking demographic methods. Rostock, Germany: Max Planck Institute for Demographic Research, 2002. (Working Paper 2002-001).

RIOS-NETO, E. L. G.; MIRANDA-RIBEIRO, A.; MIRANDA-RIBEIRO, P. Fertility differentials by education in Brazil: from the conclusion of fertility to the onset of postponement transition. Population and Development Review, v. 44, n. 3, p. 489-517, 2018.

RYDER, N. B. Problems of trend determination during a transition in fertility. Milbank Memorial Fund Quarterly, v. 34, n. 1, p. 5-21, 1956.

SIMÃO, A. B.; MIRANDA-RIBEIRO, P.; CAETANO, A. J.; CÉSAR, C. C. Comparando as idades à primeira relação sexual, à primeira união e ao nascimento do primeiro filho de duas coortes de mulheres brancas e negras em Belo Horizonte: evidências quantitativas. Revista Brasileira de Estudos de População, v. 23, n. 1, p. 151-166, 2006.

\section{Sobre os autores}

Adriana Miranda-Ribeiro é doutora em Demografia. Professora adjunta do Departamento de Demografia e pesquisadora do Centro de Desenvolvimento e Planejamento Regional (Cedeplar) da Universidade Federal de Minas Gerais (UFMG).

Ricardo Alexandrino Garcia é doutor em Demografia. Professor associado do Departamento de Geografia da Universidade Federal de Minas Gerais (UFMG).

Tereza Cristina de Azevedo Bernardes Faria é mestre em Análise e Modelagem de Sistemas Ambientais. Doutoranda em Demografia, no Centro de Desenvolvimento e Planejamento Regional (Cedeplar) da Universidade Federal de Minas Gerais (UFMG).

\section{Endereço para correspondência}

Adriana Miranda-Ribeiro

Universidade Federal de Minas Gerais, Faculdade de Ciências Econômicas, Departamento de Demografia

Av. Pres. Antônio Carlos, 6627 Pampulha

31270-901 - Belo Horizonte-MG, Brasil

Ricardo Alexandrino Garcia

Universidade Federal de Minas Gerais, Instituto de Geociências, Departamento de Geografia

Av. Pres. Antônio Carlos, 6627 Pampulha

31270-901 - Belo Horizonte-MG, Brasil 
Tereza Cristina de Azevedo Bernardes Faria

Universidade Federal de Minas Gerais, Faculdade de Ciências Econômicas, Secretaria do

Programa de Pós-Graduação em Demografia

Av. Pres. Antônio Carlos, 6627 Pampulha

31270-901 - Belo Horizonte-MG, Brasil

\section{Abstract}

Low fertility and postponement of first birth in Brazil

Brazil is undergoing a process of birth postponement, which has contributed to the reduction of observed fertility. In this context, first births are crucial, insofar as the moment of their occurrence is related to subsequent births and to postponed births' recovery potential. The objective of this paper is to analyze first-order births, taking into account Brazilian regional heterogeneity. We intend to enrich the debate about the future of fertility in Brazil. Data come from the 1980, 1991, 2000 and 2010 Demographic Censuses and the birth stories reconstructed from them, for the 1980 to 2010 period. Results show the persistence of regional differentials and point to (i) a scenario of postponement of first child in Brazil; and (ii) an increase in the proportion of childless women. If regional differences are reduced, postponement tends to increase and fertility levels tend to decrease further.

Keywords: Fertility postponement. First order births. Brazil.

\section{Resumen}

\section{Baja fecundidad y aplazamiento del primer hijo en Brasil}

Brasil pasa por un proceso de aplazamiento de los nacimientos que ha contribuido a que la fecundidad se vea reducida por la acción del efecto tiempo. En ese contexto, el primer nacimiento adquiere importancia, en la medida en que el momento de su ocurrencia está relacionado con el potencial de recuperación de los nacimientos pospuestos. El objetivo de este trabajo es analizar el comportamiento de los nacimientos de primer orden, teniendo en cuenta la heterogeneidad regional. Se buscan elementos que puedan enriquecer el debate sobre el futuro de la fecundidad en Brasil. Se utilizan los datos de los censos demográficos de 1980, 1991, 2000 y 2010 y las historias de nacimientos reconstruidas a partir de los censos para el período 1980-2010. Los resultados muestran la persistencia de las diferencias regionales y apuntan a un escenario de aplazamiento del primer hijo y de aumento de la proporción de mujeres que terminan el período reproductivo sin hijos. Si las diferencias regionales se reducen, la tendencia es de acentuación del aplazamiento y de caída adicional de los niveles de fecundidad.

Palabras clave: Aplazamiento de la fecundidad. Nacimientos de primer orden. Brasil.

Recebido para publicação em 04/05/2019 Aceito para publicação em 30/08/2019 